\title{
New Rational Homoclinic and Rogue Waves for Davey-Stewartson Equation
}

\author{
Changfu Liu, ${ }^{1}$ Chuanjian Wang, ${ }^{2}$ Zhengde Dai, ${ }^{3}$ and Jun Liu ${ }^{4}$ \\ ${ }^{1}$ School of Mathematics, Wenshan University, Wenshan 663000, China \\ ${ }^{2}$ School of Science, Kunming University of Science and Technology, Kunming 650031, China \\ ${ }^{3}$ School of Mathematics and Statistics, Yunnan University, Kunming 650091, China \\ ${ }^{4}$ Department of Mathematics and Information Science, Qujing Normal University, Qujing 655000, China
}

Correspondence should be addressed to Changfu Liu; chfuliu@163.com

Received 4 February 2014; Revised 20 March 2014; Accepted 25 March 2014; Published 16 April 2014

Academic Editor: Weiguo Rui

Copyright (C) 2014 Changfu Liu et al. This is an open access article distributed under the Creative Commons Attribution License, which permits unrestricted use, distribution, and reproduction in any medium, provided the original work is properly cited.

\begin{abstract}
A new method, homoclinic breather limit method (HBLM), for seeking rogue wave solution of nonlinear evolution equation is proposed. A new family of homoclinic breather wave solution, and rational homoclinic solution (homoclinic rogue wave) for DSI and DSII equations are obtained using the extended homoclinic test method and homoclinic breather limit method (HBLM), respectively. Moreover, rogue wave solution is exhibited as period of periodic wave in homoclinic breather wave approaches to infinite. This result shows that rogue wave can be generated by extreme behavior of homoclinic breather wave for higher dimensional nonlinear wave fields.
\end{abstract}

\section{Introduction}

In recent years, rogue waves, as a special type of solitary waves, has been triggered much interest in various physical branches, although there is no exact definition up to now. Rogue waves is a kind of waves that seems abnormal which is first observed in the deep ocean, it has been the subject of intensive research in oceanography $[1,2]$, optical fibres [3-5], superfluids [6], Bose-Einstein condensates, financial markets, and related fields [7-10]. A possible mechanism for the formation of rogue waves is associated with modulation instability [11-14].The mysteriousness of rogue wave events mainly lies in the phenomenon which appears out of nowhere and disappears without trace. As is known, there are some methods to seek rogue wave such as Darboux dressing technique, Hirota bilinear method. Based on Hirota bilinear equation of nonlinear evolution equation, for Schrödinger type complex systems, there are some effective techniques such as the Peregrine breather method ( $\mathrm{PB})$ [11], whose representation is mathematically a ratio of two polynomials, Ma solitons [4] (MS) and Akhmediev breather methods (ABs) [3]. The main difference between these methods is the test function to Hirota bilinear equation. The test functions of $\mathrm{PB}, \mathrm{MS}$, and $\mathrm{ABs}$ are $E_{1}(x, t)=e^{i \phi(x, t)}(1-((G(x, t)+i H(x, t)) / D(x, t))) ; E_{2}(x, t)=$ $e^{i \phi(x, t)}\left(\left(a_{1} \cosh (p x)+a_{2} \cos (k t)+i a_{3} \sin (k t)\right) /\left(b_{1} \cosh (p x)+\right.\right.$ $\left.\left.b_{2} \cos (k t)\right)\right)$; and $E_{3}(x, t)=e^{i \phi(x, t)}\left(\left(a_{1} \cosh (\alpha t)+a_{2} \cos (k x)\right.\right.$ $\left.\left.+i a_{3} \sinh (\alpha t)\right) /\left(b_{1} \cosh (\alpha t)+b_{2} \cos (k x)\right)\right)$, respectively. Here $\phi(x, t)$ is real function and $G(x, t), H(x, t)$, and $D(x, t)$ are polynomials of $(x, t)$, and $E_{i}(x, t), i=2,3$, may generate the rogue wave similar to $E_{1}$ as $k \rightarrow 0$. The above three methods have been successfully applied to complex system such as Hirota equation, Sasa-Satsuma equation, Davey-Stewartson equation, coupled Gross-Pitaevskii equation, coupled NLS Maxwell-Bloch equation, and coupled Schrödinger-Boussinesq equation [11-18].

In this work, we propose a homoclinic (heteroclinic) breather limit method for seeking rogue wave solution. We take $E(x, t)=e^{i \phi(x, t)}\left(\left(e^{-p(x-\alpha t)}+a_{1} \cos \left(p_{1}(x-\beta t)\right)+\right.\right.$ $\left.\left.a_{2} e^{p(x-\alpha t)}\right) /\left(e^{-p(x-\alpha t)}+a_{3} \cos \left(p_{1}(x-\beta t)\right)+a_{4} e^{p(x-\alpha t)}\right)\right)$ as a test function to Hirots bilinear equation. $E(x, t)$ can generate one or two rogue waves as $p_{1} \rightarrow 0$. It is obvious that the $E$ is different from $E_{2}$ and $E_{3}$, comparing with $E_{2}, E_{3}$, and $E$ has more complicated structure. Now we consider the application of HBLM to Davey-Stewartson equation. 
Davey-Stewartson (DS) equation is written as [19]

$$
\begin{gathered}
i u_{t}=-u_{x x}-\frac{1}{\alpha_{0}^{2}} u_{y y}-\frac{2 \epsilon}{\alpha_{0}^{2}}|u|^{2} u-\frac{2}{\alpha_{0}^{2}} u v, \\
v_{y y}-\alpha_{0}^{2} v_{x x}-2 \alpha_{0}^{2} \epsilon\left(|u|^{2}\right)_{x x}=0,
\end{gathered}
$$

where $u: R_{x} \times R_{y} \times R_{t}^{+} \rightarrow C, v: R_{x} \times R_{y} \times R_{t}^{+} \rightarrow R$, and $\epsilon$ and $\alpha_{0}$ are constants. DS equation was introduced in a paper by Davey and Stewartson (1974) to describe the evolution of a three-dimensional disturbance in the nonlinear regime of plane Poiseuille flow. The function $u(x, y, t)$ stands for the complex amplitude, and $v(x, y, t)$ describes the perturbation of the real velocity. DS equation is called the DSI as $\epsilon=1$, $\alpha_{0}= \pm 1$ and DSII as $\epsilon=1, \alpha_{0}= \pm i$. There are known results due to local well-posed, global existence and blowup of some solutions, exact periodic soliton solutions, solitoff and dromion solutions [20-29]. Recently, homoclinic and heteroclinic tube solutions were obtained [29-33].

We consider DSI equation:

$$
\begin{aligned}
i u_{t}+u_{x x}+u_{y y} & =-2|u|^{2} u-2 u v, \\
v_{x x}-v_{y y} & =-2\left(|u|^{2}\right)_{x x}
\end{aligned}
$$

and DSII equation:

$$
\begin{aligned}
i U_{t}+U_{x x}-U_{y y} & =2|U|^{2} U+2 U V, \\
V_{x x}+V_{y y} & =-2\left(|U|^{2}\right)_{x x} .
\end{aligned}
$$

\section{Homoclinic Breather and Rogue Wave Solution of DSI}

Making transformation $u=(a / \sqrt{2}) \exp \left(i a^{2} t\right) Q, v=-\varphi / 2$ and substituting it into (2), we can get

$$
\begin{aligned}
i Q_{t}+Q_{x x}+Q_{y y} & =-a^{2}\left(|Q|^{2}-1\right) Q+Q \varphi, \\
\varphi_{x x}-\varphi_{y y} & =2 a^{2}\left(|Q|^{2}\right)_{x x},
\end{aligned}
$$

where $Q=Q(x, y, t)$ is a complex function and $\varphi$ is a real. By the dependent variable transformation

$$
Q=\frac{G}{F}, \quad \varphi=-4(\ln F)_{x x}
$$

with $G$ being a complex and $F$ being a real, then (4) can be converted into the form

$$
\begin{gathered}
i G_{t} F-i F_{t} G+G_{x x} F-2 G_{x} F_{x}+G F_{x x}+G_{y y} F \\
-2 G_{y} F_{y}+G F_{y y}-\left(a^{2}+B\right) G F=0, \\
2\left(F_{y y} F-F_{y}^{2}-F_{x x} F+F_{x}^{2}\right)-B F^{2}-a^{2} G G^{*}=0,
\end{gathered}
$$

where $B$ is an integration constant and an asterisk denotes the complex conjugation.
By means of the extended homoclinic test approach [33], we take the test function as follows:

$$
\begin{aligned}
G= & e^{-p(x+y / 2+\alpha t)}+a_{1} \cos \left(p_{1}(x+2 y-\alpha t)\right) \\
& +a_{2} e^{p(x+y / 2+\alpha t)}, \\
F= & e^{-p(x+y / 2+\alpha t)}+a_{3} \cos \left(p_{1}(x+2 y-\alpha t)\right) \\
& +a_{4} e^{p(x+y / 2+\alpha t)}
\end{aligned}
$$

where all of $a_{3}, a_{4}, p, p_{1}, \beta, \beta_{1}$, and $\alpha$ are real and $a_{1}, a_{2}$ are complex. Substituting (7) into (6) and equating the coefficients of all powers of $e^{j p(x+y / 2+\alpha t)} \cos \left(p_{1}(x+2 y-\right.$ $\alpha t)), e^{j p(x+y / 2+\alpha t)} \sin \left(p_{1}(x+2 y-\alpha t)\right)$ and $e^{ \pm 2(p(x+y / 2+\alpha t))}(j=$ $0, \pm 1)$ to zero, we can obtain a set of algebraic equations for $p, p_{1}, \beta, \beta_{1}, \alpha$, and $a_{j}, j=1,2,3,4$, with

$$
\begin{gathered}
B=-a^{2}, \quad\left(4 p p_{1}-i p_{1} \alpha\right) a_{1}+\left(4 p p_{1}+i p_{1} \alpha\right) a_{3}=0 \\
\left(-i p_{1} \alpha-4 p p_{1}\right) a_{4} a_{1}+\left(i p_{1} \alpha-4 p p_{1}\right) a_{3} a_{2}=0 \\
\left(i p \alpha+\frac{5 p^{2}}{4}-5 p_{1}^{2}\right) a_{1}+\left(\frac{5 p^{2}}{4}-5 p_{1}^{2}-i p \alpha\right) a_{3}=0 \\
\left(\frac{5 p^{2}}{4}-i p \alpha-5 p_{1}^{2}\right) a_{4} a_{1}+\left(i p \alpha+\frac{5 p^{2}}{4}-5 p_{1}^{2}\right) a_{3} a_{2}=0 \\
-10 a_{1} a_{3} p_{1}^{2}+\left(5 p^{2}+2 i p \alpha\right) a_{2}+\left(5 p^{2}-2 i p \alpha\right) a_{4}=0 \\
a^{2}\left(a_{3}^{2}-a_{1} a_{1}^{*}\right)=0, \quad a^{2}\left(a_{4}^{2}-a_{2} a_{2}^{*}\right)=0 \\
\left(2 a^{2}-\frac{3 p^{2}}{2}-6 p_{1}^{2}\right) a_{3}-a^{2}\left(a_{1}+a_{1}^{*}\right)=0 \\
\left(2 a^{2}-\frac{3 p^{2}}{2}-6 p_{1}^{2}\right) a_{3} a_{4}-a^{2}\left(a_{1} a_{2}^{*}+a_{1}^{*} a_{2}\right)=0 \\
\left(2 a^{2}-6 p^{2}\right) a_{4}-6 a_{3}^{2} p_{1}^{2}-a^{2}\left(a_{2}+a_{2}^{*}\right)=0
\end{gathered}
$$

Solving these equations, we obtain the relations between the parameters as

$$
\begin{gathered}
B=-a^{2}, \quad p_{1}^{2}=\frac{21 p^{2}}{20}, \\
p^{2}=\frac{320 a^{2}-39 \alpha^{2}}{624}, \quad a_{1}=\frac{(i \alpha+4 p) a_{3}}{i \alpha-4 p}, \\
a_{2}=\frac{(i \alpha+4 p)^{2} a_{4}}{(i \alpha-4 p)^{2}}, \quad a_{3}^{2}=\frac{4\left(21 \alpha^{2}-80 p^{2}\right) a_{4}}{21\left(\alpha^{2}+16 p^{2}\right)} .
\end{gathered}
$$


From $p^{2} \geq 0$ and $a_{3}^{2} \geq 0$ in (9), we have $\left(800 a^{2} / 507\right) \leq$ $\alpha^{2} \leq\left(320 a^{2} / 39\right)$. Substituting (9) into (7) and then (5) and taking $a_{4}>0$, we obtain the solution for DSI equation as

$$
\begin{aligned}
u=\frac{a}{\sqrt{2}} & e^{i\left(\theta+a^{2} t\right)} \frac{2 \cosh [\xi+\gamma+i \theta]+\left(a_{3} / \sqrt{a_{4}}\right) \cos (\eta)}{2 \cosh (\xi+\gamma)+\left(a_{3} / \sqrt{a_{4}}\right) \cos (\eta)}, \\
v=2( & 2 a_{3} \sqrt{a_{4}}\left(p^{2}-p_{1}^{2}\right) \cos (\eta) \cosh (\xi+\gamma) \\
& +4 a_{3} p p_{1} \sqrt{a_{4}} \sin (\eta) \sinh (\xi+\gamma) \\
& \left.+4 a_{4} p^{2}-a_{3}^{2} p_{1}^{2}\right) \\
\times & \left(\left[a_{3} \cos (\eta)+2 \sqrt{a_{4}} \cosh (\xi+\gamma)\right]^{2}\right)^{-1},
\end{aligned}
$$

where $\xi=p(x+y / 2+\alpha t), \eta=p_{1}(x+2 y-\alpha t), \gamma=\ln \sqrt{a_{4}}$, $e^{i \theta}=(i \alpha+4 p) /(i \alpha-4 p)$, and $p, p_{1}, \alpha, a_{3}$, and $a_{4}$, are given by (9). Note that if $(u(x, y, t), v(x, y, t))$ is the solution of DSI equation, then $(u(x,-y, t), v(x,-y, t))$ is the solution as well. So, we also obtain solution of DSI equation:

$$
\begin{aligned}
u_{1}=\frac{a}{\sqrt{2}} & e^{i\left(\theta+a^{2} t\right)} \frac{2 \cosh \left[\xi_{1}+\gamma+i \theta\right]+\left(a_{3} / \sqrt{a_{4}}\right) \cos \left(\eta_{1}\right)}{2 \cosh \left(\xi_{1}+\gamma\right)+\left(a_{3} / \sqrt{a_{4}}\right) \cos \left(\eta_{1}\right)}, \\
v_{1}=2( & 2 a_{3} \sqrt{a_{4}}\left(p^{2}-p_{1}^{2}\right) \cos \left(\eta_{1}\right) \cosh \left(\xi_{1}+\gamma\right) \\
& +4 a_{3} p p_{1} \sqrt{a_{4}} \sin \left(\eta_{1}\right) \sinh \left(\xi_{1}+\gamma\right) \\
& \left.+4 a_{4} p^{2}-a_{3}^{2} p_{1}^{2}\right) \\
& \times\left(\left[a_{3} \cos \left(\eta_{1}\right)+2 \sqrt{a_{4}} \cosh \left(\xi_{1}+\gamma\right)\right]^{2}\right)^{-1},
\end{aligned}
$$

where $\xi_{1}=p(x-y / 2+\alpha t), \eta_{1}=p_{1}(x-2 y-\alpha t)$, and $\gamma=\ln \sqrt{a_{4}}$. Solution (11) is the homoclinic solution of DSI equation. Indeed, we have

$$
\begin{gathered}
\left(u_{1}, v_{1}\right) \longrightarrow\left(\frac{a}{\sqrt{2}} \exp \left(i\left(a^{2} t+2 \theta\right)\right), 0\right), \\
\text { as } t \longrightarrow+\infty ; \\
\left(u_{1}, v_{1}\right) \longrightarrow\left(\frac{a}{\sqrt{2}} \exp \left(i a^{2} t\right), 0\right), \quad \text { as } t \longrightarrow-\infty,
\end{gathered}
$$

where $2 \theta$ is a phase shift and $\left(a \exp \left(i a^{2} t\right), 0\right)$ is a fixed circle of DSI [30]. Note that solution (11) contains not only a periodic wave $\cos \left(p_{1}(x-2 y-\alpha t)\right)$, so its amplitude periodically oscillates with the evolution of time (the breather effect), but also a solitary wave $1 / \cosh (p(x-y / 2+\alpha t)+\gamma)$, which shows that interaction between a solitary wave and a periodic wave with the same velocity $\alpha$ and opposite propagation direction can form a new family of homoclinic solution. This is a new phenomenon of evolution of a three-dimensional disturbance in the nonlinear regime of plane Poiseuille flow (Figure 1).

In the above two cases, set $a_{3}=-(2)$ 21) $\sqrt{\left(441 r^{2}-1680 p^{2}\right) /\left(16 p^{2}+r^{2}\right)}$ and $a_{4}=1$, when $\alpha=(8 / 39) \sqrt{195} a$. Let $p \rightarrow 0$, and we can obtain two rogue wave solutions for DSI as follows (Figures 2 and 3 ):

$$
\begin{aligned}
& u_{1}=\frac{a}{\sqrt{2}} \exp \left(i a^{2} t\right) \\
& \times\left(\left(861 \alpha^{4} t^{2}+(-1344 y t-42 x t) \alpha^{3}\right.\right. \\
& +\left(2184 x y+861 x^{2}+1869 y^{2}\right) \alpha^{2} \\
& -18560) \\
& \times\left(861 \alpha^{4} t^{2}+(-1344 y t-42 x t) \alpha^{3}\right. \\
& +\left(2184 x y+861 x^{2}+1869 y^{2}\right) \alpha^{2} \\
& +8320)^{-1} \\
& +i\left(-6720 \alpha^{2} t+(-3360 y-6720 x) \alpha\right) \\
& \times\left(861 \alpha^{4} t^{2}+(-1344 y t-42 x t) \alpha^{3}\right. \\
& +\left(2184 x y+861 x^{2}+1869 y^{2}\right) \alpha^{2} \\
& \left.+8320)^{-1}\right) \text {, } \\
& v_{1}=-84 \alpha^{2}\left(-35259 \alpha^{4} t^{2}\right. \\
& +(50736 y t-1722 x t) \alpha^{3} \\
& +\left(35301 x^{2}+89544 x y+36939 y^{2}\right) \alpha^{2} \\
& -341120) \\
& \times\left(\left(861 \alpha^{4} t^{2}+(-1344 y t-42 x t) \alpha^{3}\right.\right. \\
& +\left(2184 x y+861 x^{2}+1869 y^{2}\right) \alpha^{2} \\
& \left.+8320)^{2}\right)^{-1}, \\
& u_{2}=\frac{a}{\sqrt{2}} \exp \left(i a^{2} t\right) \\
& \times\left(\left(861 \alpha^{4} t^{2}+(1344 y t-42 x t) \alpha^{3}\right.\right. \\
& +\left(-2184 x y+861 x^{2}+1869 y^{2}\right) \alpha^{2} \\
& -18560) \\
& \times\left(861 \alpha^{4} t^{2}+(1344 y t-42 x t) \alpha^{3}\right. \\
& +\left(-2184 x y+861 x^{2}+1869 y^{2}\right) \alpha^{2} \\
& +8320)^{-1} \\
& +i\left(-6720 \alpha^{2} t+(3360 y-6720 x) \alpha\right)
\end{aligned}
$$




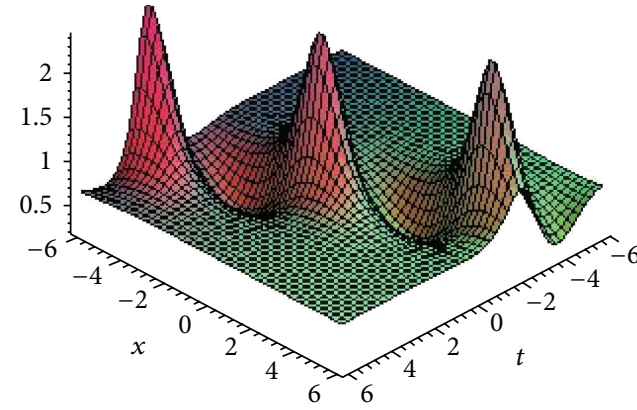

(a)

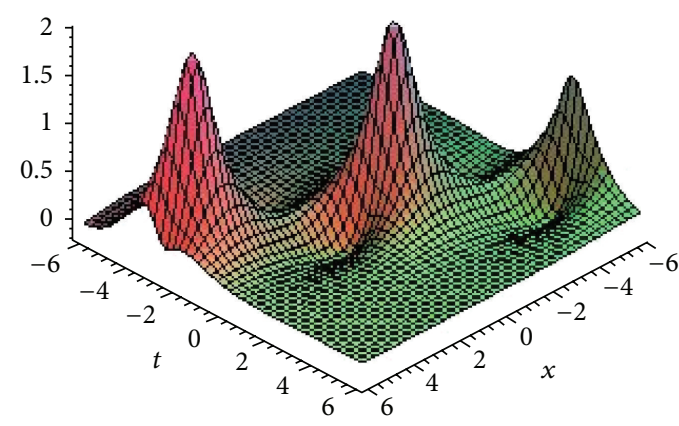

(b)

FIgURE 1: (a) Homoclinic breather wave of $\left|u_{1}\right|$ in DSI. (b) Homoclinic breather wave of $v_{1}$ in DSI.

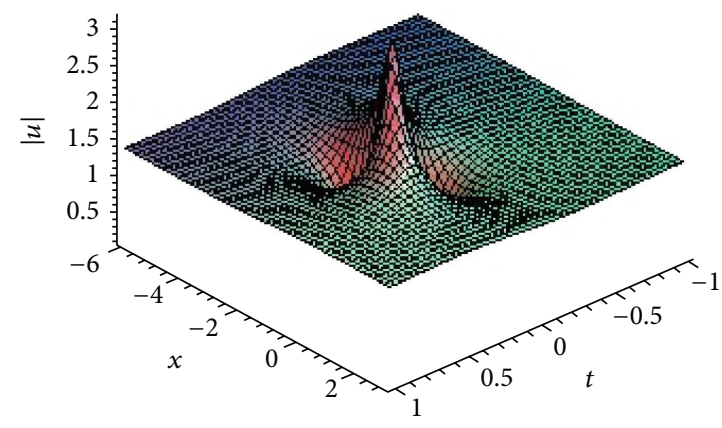

(a)

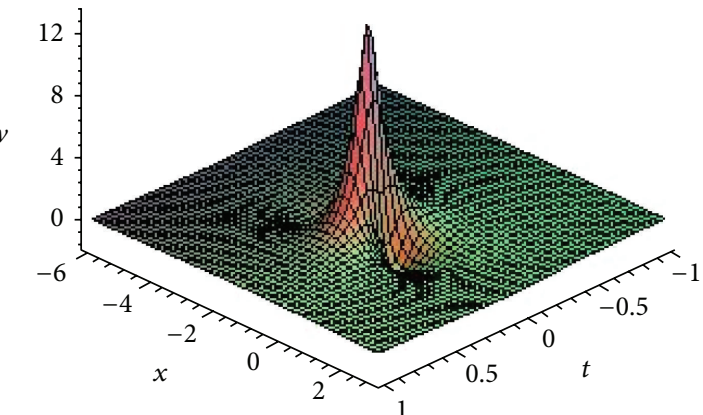

(b)

Figure 2: (a) Homoclinic rogue wave $\left|u_{1}\right|$ in solution (13). (b) Homoclinic rogue wave $v_{1}$ in solution (13).

$$
\begin{aligned}
& \times( 861 \alpha^{4} t^{2}+(1344 y t-42 x t) \alpha^{3} \\
&+\left(-2184 x y+861 x^{2}+1869 y^{2}\right) \alpha^{2} \\
&+\left.8320)^{-1}\right) \\
& v_{2}=-84 \alpha^{2}\left(-35259 \alpha^{4} t^{2}\right. \\
&+(-50736 y t-1722 x t) \alpha^{3} \\
&+\left(35301 x^{2}-89544 x y+36939 y^{2}\right) \alpha^{2} \\
&\quad-341120) \\
& \times\left(\left(861 \alpha^{4} t^{2}+(-1344 y t-42 x t) \alpha^{3}\right.\right. \\
&+\left(-2184 x y+861 x^{2}+1869 y^{2}\right) \alpha^{2} \\
&\left.+8320)^{2}\right)^{-1},
\end{aligned}
$$

where $\alpha=(8 / 39) \sqrt{195} a$.

Both $\left(u_{1}, v_{1}\right)$ and $\left(u_{2}, v_{2}\right)$ are rational homoclinic (rogue) wave solutions of DSI equation. In fact, we have $\left(u_{j}, v_{j}\right) \rightarrow$ $\left((a / \sqrt{2}) \exp \left(i a^{2} t\right), 0\right)$ as $t \rightarrow \infty, j=1,2$.

\section{Homoclinic Breather and Rogue Wave Solution of DSII}

As we know, the $\left(a e^{-2 a^{2} i t}, 0\right)$ is hyperbolic fixed cycle of DSII equation when the period of $y$ is larger than the period of $x$ [30]. Similar to the argument in [30], we can analyze the linear stability of fixed cycle $\left(a e^{-2|a|^{2} i t}, 0\right)$. Similar to the dealing with process of (2), by means of transformation of functions

$$
U=\frac{G}{F}, \quad V=-2(\ln F)_{x x},
$$

Equation (3) can be converted into the bilinear form

$$
\begin{gathered}
\left(i D_{t}+D_{x}^{2}-D_{y}^{2}\right) G \cdot F=\lambda G \cdot F \\
\left(D_{x}^{2}+D_{y}^{2}+\lambda\right) F \cdot F=2 G G^{*},
\end{gathered}
$$

where $G$ is a complex function and $F$ is a real. Now, we take the following ansatz:

$$
\begin{aligned}
G= & a e^{-2 a^{2} i t}\left[e^{-p_{2}(m x+n y+\alpha t)}+b_{1} \cos p_{1}(k x+l y-\alpha t)\right. \\
& \left.+b_{2} e^{p_{2}(m x+n y+\alpha t)}\right] \\
F= & e^{-p_{2}(m x+n y+\alpha t)}+b_{3} \cos p_{1}(k x+l y-\alpha t) \\
& +b_{4} e^{p_{2}(m x+n y+\alpha t)}
\end{aligned}
$$




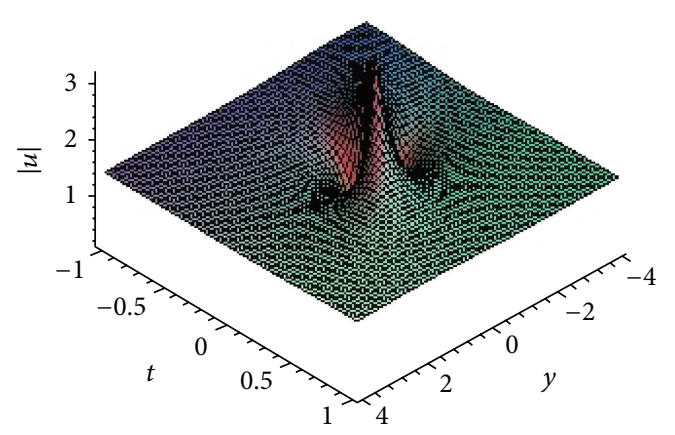

(a)

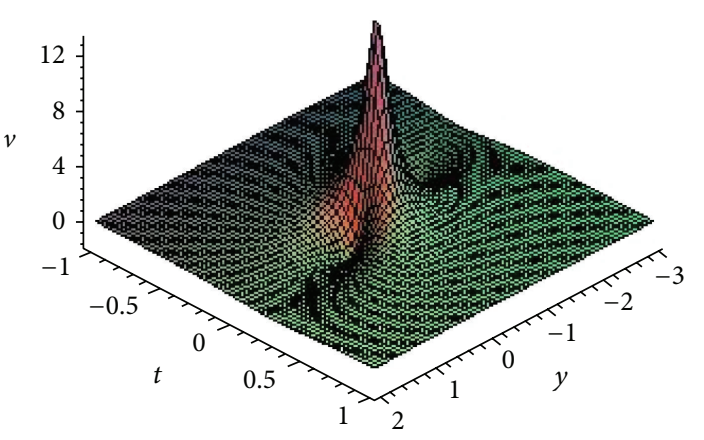

(b)

Figure 3: (a) Behaviour of $\left|u_{2}\right|$ in solution (14). (b) Behaviour of $v_{2}$ in solution (14).

where $p_{1}, p_{2}, m, n, k, l, \alpha, b_{3}$, and $b_{4}$ are real and $b_{1}, b_{2}$ are complex. Substituting (17) into (16), then we obtain

$$
\begin{gathered}
\lambda=2 a^{2}, \\
p_{2}^{2}=\left(8\left(k^{2}-l^{2}\right)(k m-n l)^{2} a^{2}\right. \\
+\left(l^{3} n-\left(k m+m^{2}\right) l^{2}\right. \\
\left.\left.+k^{2} n l+n^{2} k^{2}-k^{3} m\right) \alpha^{2}\right) \\
\times\left(4 \left(k^{3} m-k^{2} n l-n^{2} k^{2}\right.\right. \\
\left.+l^{2} k m+m^{2} l^{2}-l^{3} n\right) \\
\left.\times(k m-n l)^{2}\right)^{-1}, \\
p_{1}^{2}=\frac{\left(m^{2}-n^{2}-2 n l+2 k m\right) p_{2}^{2}}{k^{2}-l^{2}}, \\
b_{1}=\frac{b_{3}\left(i \alpha+2 p_{2} m k-2 p_{2} n l\right)}{i \alpha-2 p_{2} m k+2 p_{2} n l}, \\
\times\left(\left(2 m k-n^{2}-2 n l+m^{2}\right)\right. \\
b_{2}=\frac{b_{4}\left(i \alpha+2 p_{2} m k-2 p_{2} n l\right)^{2}}{\left(i \alpha-2 p_{2} m k+2 p_{2} n l\right)^{2}}, \\
\left.\left.\times\left(4 m^{2} k^{2}-8 n l m k+4 n^{2} l^{2}\right) p_{2}^{2}+\alpha^{2}\right)\right) \\
-4 b_{4} \quad n l+k m=0 . \\
\times\left(m^{2}-n^{2}\right)(m k-n l)^{2} p_{2}^{2} \\
\left.\left(2 m k-n^{2}-2 n l+m^{2}\right)\right)
\end{gathered}
$$

So we can obtain breather solution of DSII equation as follows:

$$
\begin{aligned}
& U= a e^{-i\left(2 a^{2} t-\theta\right)} \\
& \times\left(2 \sqrt{b_{4}} \cosh \left(p_{2}(m x+n y+\alpha t)+i \theta+\phi\right)\right. \\
&\left.+b_{3} \cos \left(p_{1}(k x+l y-\alpha t)\right)\right) \\
& \times\left(2 \sqrt{b_{4}} \cosh \left(p_{2}(m x+n y+\alpha t)+\phi\right)\right. \\
&\left.+b_{3} \cos \left(p_{1}(k x+l y-\alpha t)\right)\right)^{-1} \\
&-2( 2 \sqrt{b_{4}} \\
& \times \cosh \left(p_{2}(m x+n y+\alpha t)+\phi\right) p_{2}^{2} m^{2} \\
&\left.-b_{3} \cos \left(p_{1}(k x+l y-\alpha t)\right) p_{1}^{2} k^{2}\right) \\
& \times\left(2 \sqrt{b_{4}} \cosh \left(p_{2}(m x+n y+\alpha t)+\phi\right)\right. \\
&+\left.b_{3} \cos \left(p_{1}(k x+l y-\alpha t)\right)\right)^{-1} \\
&+2(\left(2 \sqrt{b_{4}} \sinh \left(p_{2}(m x+n y+\alpha t)+\phi\right) p_{2} m\right. \\
& \times\left.\left.+b_{3} \cos \left(p_{1}(k x+l y-\alpha t)\right)\right)^{2}\right) \\
&\left.\left.-b_{3} \sin \left(p_{1}(k x+l y-\alpha t)\right) p_{1} k\right)^{2}\right) \\
& b_{4} \cosh \left(p_{2}(m x+n y+\alpha t)+\phi\right)
\end{aligned}
$$

where $\theta=\arctan b_{1}$ and $\phi=\ln \left(\sqrt{b_{4}}\right)$. 


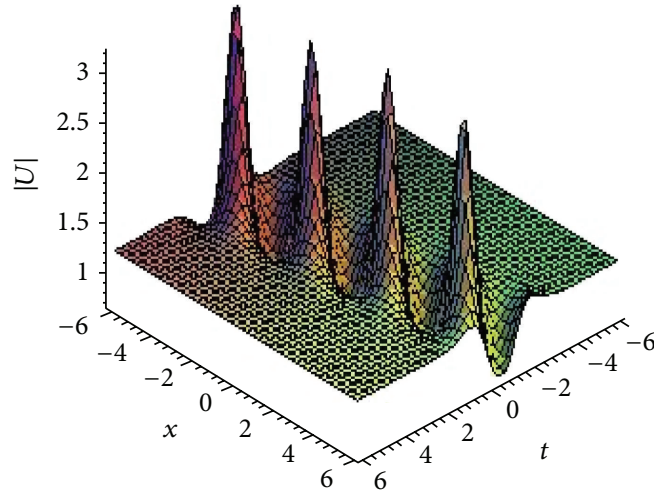

(a)

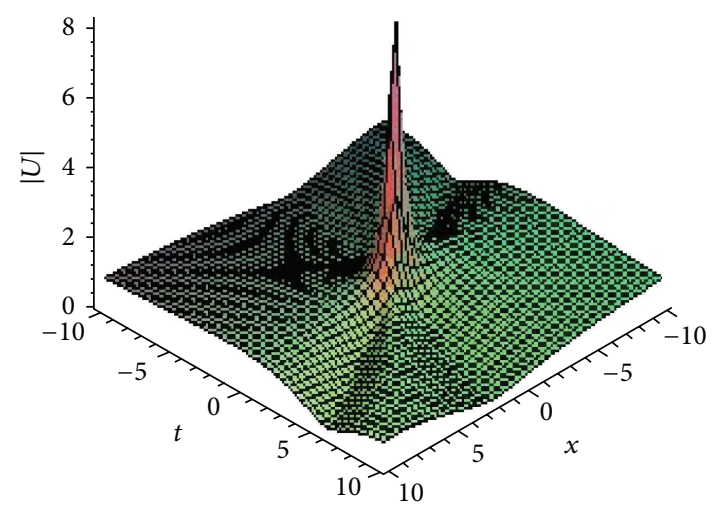

(b)

FIgURE 4: (a) Bright breather structure $|U|$ in DSII. (b) Bright rogue wave $|U|$ in DSII.

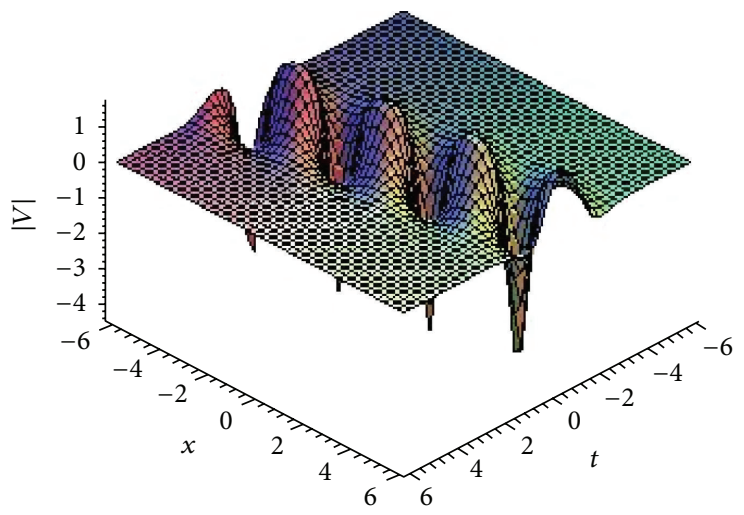

(a)

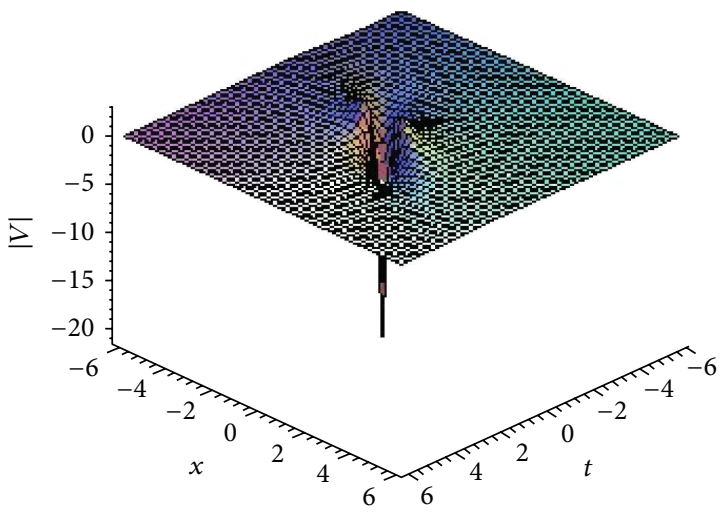

(b)

FIgURE 5: (a) Dark breather structure $V$ in DSII. (b) Dark rogue wave $V$ in DSII.

The rogue wave of the DSII system is derived when the period of periodic wave goes to infinite. Indeed, by letting $p_{1} \rightarrow 0$ in solution (19), solution (19) becomes rogue waves:

$$
\begin{aligned}
U= & a e^{2 i a^{2} t} \\
\times & \left(-(m x+n y+\alpha t)^{2}+2(k x+l y-\alpha t)^{2}\right. \\
& \left.+16 i(m x+n y+\alpha t) \delta_{1} \delta_{2}+\delta_{4}\right) \\
\times & \left(2(m x+n y+\alpha t)^{2} \delta_{1}\right. \\
& \left.+2(k x+l y-\alpha t)^{2}+\delta_{3}\right)^{-1} \\
V= & 2\left(4 m^{2} \delta_{1}+4 k^{2}\right) \\
\times & \left(2(m x+n y+\alpha t)^{2} \delta_{1}\right. \\
& \left.+2(k x+l y-\alpha t)^{2}+\delta_{3}\right)^{-1}
\end{aligned}
$$

$$
\begin{gathered}
+2\left(4(m x+n y+\alpha t) \delta_{1} m\right. \\
+4(k x+l y-\alpha t) k)^{2} \\
\times\left(\left(2(m x+n y+\alpha t)^{2} \delta_{1}\right.\right. \\
\left.\left.+2(k x+l y-\alpha t)^{2}+\delta_{3}\right)^{2}\right)^{-1},
\end{gathered}
$$

where

$$
\begin{aligned}
& \delta_{1}=\frac{k^{2}-l^{2}}{m^{2}-n^{2}-2 \ln +2 m k}, \\
& \delta_{2}=\frac{m k-\ln }{\alpha}, \\
& \delta_{3}=16 \frac{\left(k^{2}-l^{2}\right)\left(m k+m^{2}-n^{2}-\ln \right)(m k-\ln )^{2}}{\left(m^{2}-n^{2}-2 \ln +2 m k\right)^{2} \alpha^{2}},
\end{aligned}
$$




$$
\begin{aligned}
\delta_{4} & =32 \frac{\left(k^{2}-l^{2}\right)\left(5 m k+3 m^{2}-5 \ln -3 n^{2}\right)(m k-l n)^{2}}{\left(m^{2}-n^{2}-2 \ln +2 m k\right)^{2} \alpha^{2}}, \\
m & =\frac{1312 \alpha^{2}}{9\left(41 \alpha^{2}+512 a^{2}\right)}, \\
n & =-\frac{1640 \alpha^{2}}{9\left(41 \alpha^{2}+512 a^{2}\right)} .
\end{aligned}
$$

Figure 4(a): The dynamical evolution of bright breather $|U(x, t)|$ in solution (19) is plotted with parameters $a=\alpha=$ $k=2, b_{4}=1$, and $l=1.6$.

Figure 4(b): The dynamical evolution of bright rogue wave $|U(x, t)|$ in solution (20) is plotted with parameters $a=$ $\alpha=k=2, b_{4}=1$, and $l=1.6$.

Figure 5(a): The dynamical evolution of dark breather $V(x, t)$ in solution (19) is plotted with parameters $a=\alpha=$ $k=2, b_{4}=1$, and $l=1.6$.

Figure 5(b): The dynamical evolution of dark rogue wave $V(x, t)$ in solution $(20)$ is plotted with parameters $a=\alpha=$ $k=2, b_{4}=1$, and $l=1.6$.

It is easy to verify that both solution (19) and (20) are solutions of DSII. Similar to DSI, we can show that solution (20) is a rational homoclinic rogue waves.

\section{Conclusion}

In summary, based on Hirota bilinear form, applying homoclinic breather limit method to DSI and DSII equations, we obtain a new kind of homoclinic solutions with locally oscillatory structure and rational homoclinic rogue wave solutions. We also investigate and exhibit the different homoclinic rogue wave structures of solutions. These results show the complexity and variety of dynamical behavior of the DS system. Following these ideas in this work, the problem needed to further study is other types of nonlinear evolution equations whether have this kind of rational homoclinic solutions.

\section{Conflict of Interests}

The authors declare that there is no conflict of interests regarding the publication of this paper.

\section{Acknowledgment}

This work was supported by Chinese Natural Science Foundation Grant nos. 11261049 and 11361048.

\section{References}

[1] M. Hopkin, "Sea snapshots will map frequency of freak waves," Nature, vol. 430, no. 6999, p. 492, 2004.

[2] C. Kharif, E. Pelinovsky, and A. Slunyaev, Rogue Waves in the Ocean: Observation, Theories and Modeling, Springer, New York, NY, USA, 2009.
[3] N. Akhmediev, A. Ankiewicz, and J. M. Soto-Crespo, "Rogue waves and rational solutions of the nonlinear Schrödinger equation," Physical Review E, vol. 80, no. 2, Article ID 026601, 9 pages, 2009.

[4] Y. C. Ma, "The perturbed plane-wave solutions of the cubic Schrödinger equation," Studies in Applied Mathematics, vol. 60, no. 1, pp. 43-58, 1979.

[5] D. R. Solli, C. Ropers, P. Koonath, and B. Jalali, "Optical rogue waves," Nature, vol. 450, no. 7172, pp. 1054-1057, 2007.

[6] Y. V. Bludov, V. V. Konotop, and N. Akhmediev, "Rogue waves as spatial energy concentrators in arrays of nonlinear waveguides," Optics Letters, vol. 34, no. 19, pp. 3015-3017, 2009.

[7] A. N. Ganshin, V. B. Efimov, G. V. Kolmakov, L. P. MezhovDeglin, and P. V. E. McClintock, "Observation of an inverse energy cascade in developed acoustic turbulence in superfluid helium," Physical Review Letters, vol. 101, no. 6, Article ID 065303, 4 pages, 2008.

[8] Y. V. Bludov, V. V. Konotop, and N. Akhmediev, "Matter rogue waves," Physical Review A: Atomic, Molecular, and Optical Physics, vol. 80, no. 3, Article ID 033610, 5 pages, 2009.

[9] A. Montina, U. Bortolozzo, S. Residori, and F. T. Arecchi, "Nongaussian statistics and extreme waves in a nonlinear optical cavity," Physical Review Letters, vol. 103, no. 17, Article ID 173901, 4 pages, 2009.

[10] Z. Y. Yan, "Financial rogue waves," Communications in Theoretical Physics, vol. 54, no. 5, pp. 947-949, 2010.

[11] D. H. Peregrine, "Water waves, nonlinear Schrödinger equations and their solutions," Australian Mathematical Society B: Applied Mathematics, vol. 25, no. 1, pp. 16-43, 1983.

[12] Y. Tao and J. He, "Multisolitons, breathers, and rogue waves for the Hirota equation generated by the Darboux transformation," Physical Review E, vol. 85, no. 2, Article ID 026601, 7 pages, 2012.

[13] U. Bandelow and N. Akhmediev, "Persistence of rogue waves in extended nonlinear Schrödinger equations: Integrable SasaSatsuma case," Physics Letters A: General, Atomic and Solid State Physics, vol. 376, no. 18, pp. 1558-1561, 2012.

[14] Y. Ohta and J. Yang, "Dynamics of rogue waves in the DaveyStewartson II equation," Journal of Physics A: Mathematical and Theoretical, vol. 46, no. 10, Article ID 105202, 19 pages, 2013.

[15] L. C. Zhao and J. Liu, "Rogue-wave solutions of a threecomponent coupled nonlinear Schrödinger equation," Physical Review E, vol. 87, no. 1, Article ID 013201, 8 pages, 2013.

[16] W. P. Zhong, "Rogue wave solutions of the generalized onedimensional Gross-Pitaevskii equation," Journal of Nonlinear Optical Physics \& Materials, vol. 21, no. 2, Article ID 1250026, 9 pages, 2012.

[17] C. Li, J. He, and K. Porseizan, "Rogue waves of the Hirota and the Maxwell-Bloch equations," Physical Review E, vol. 87, no. 1, Article ID 012913, 13 pages, 2013.

[18] G. Mu and Z. Qin, "Rogue waves for the coupled SchrödingerBoussinesq equation and the coupled Higgs equation," Journal of the Physical Society of Japan, vol. 81, no. 8, Article ID 084001, 6 pages, 2012.

[19] A. Davey and K. Stewartson, "On three-dimensional packets of surface waves," Proceedings of the Royal Society A: Mathematical, Physical \& Engineering Sciences, vol. 338, pp. 101-110, 1974.

[20] V. A. Arkadiev, A. K. Pogrebkov, and M. C. Polivanov, "Inverse scattering transform method and soliton solutions for DaveyStewartson II equation," Physica D: Nonlinear Phenomena, vol. 36, no. 1-2, pp. 189-197, 1989. 
[21] M. Boiti, J. J. Leon, L. Martina, and F. Pempinelli, "Scattering of localized solitons in the plane," Physics Letters A, vol. 132, no. 8-9, pp. 432-439, 1988.

[22] M. Boiti, J. P. Leon, and F. Pempinelli, "Multidimensional solitons and their spectral transforms," Journal of Mathematical Physics, vol. 31, no. 11, pp. 2612-2618, 1990.

[23] K. W. Chow, "A class of doubly periodic waves for nonlinear evolution equations," Wave Motion, vol. 35, no. 1, pp. 71-90, 2002.

[24] A. S. Fokas and P. M. Santini, "Coherent structures in multidimensions," Physical Review Letters, vol. 63, no. 13, pp. 1329-1333, 1989.

[25] C. R. Gilson, "Resonant behaviour in the Davey-Stewartson equation," Physics Letters. A, vol. 161, no. 5, pp. 423-428, 1992.

[26] J. Hietarinta and R. Hirota, "Multidromion solutions to the Davey-Stewartson equation," Physics Letters A, vol. 145, no. 5, pp. 237-244, 1990.

[27] J. Satsuma and M. J. Ablowitz, "Two-dimensional lumps in nonlinear dispersive systems," Journal of Mathematical Physics, vol. 20, no. 7, pp. 1496-1503, 1979.

[28] M. Tajiri and T. Arai, "Periodic soliton solutions to the DaveyStewartson equation," Proceedings of Institute of Mathematics of NAS of Ukraine, vol. 30, part 1, pp. 210-217, 2000.

[29] Y. Watanabe and M. Tajiri, "Periodic soliton resonance: solutions to the Davey-Stewartson I equation," Journal of the Physical Society of Japan, vol. 67, no. 3, pp. 705-708, 1998.

[30] Z. Dai and J. Huang, "Homoclinic tubes for the DaveyStewartson II equation with periodic boundary conditions," Chinese Journal of Physics, vol. 43, no. 2, pp. 349-356, 2005.

[31] J. Huang and Z. Dai, "Homoclinic solutions for DaveyStewartson equation," Chaos, Solitons \& Fractals, vol. 35, no. 5, pp. 996-1002, 2008.

[32] Z. D. Dai, A. J. Zhu, S. L. Li, and D. L. Li, "Homoclinic tubes for the Davey-Stewartson II equation with periodic boundary conditions," Chinese Journal of Physics, vol. 45, no. 1, pp. 62-71, 2007.

[33] L. B. Santos, M. Rojas-Medar, and G. Ruiz-Garzón, "Existence of weakly efficient solutions in vector optimization," Acta Mathematica Sinica, vol. 24, no. 4, pp. 599-606, 2008. 


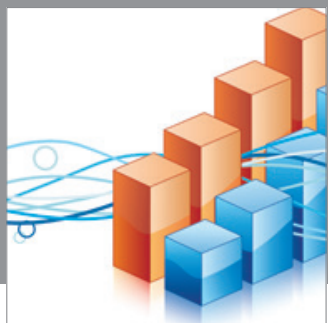

Advances in

Operations Research

mansans

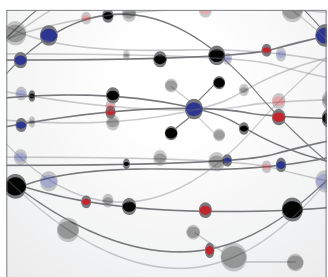

The Scientific World Journal
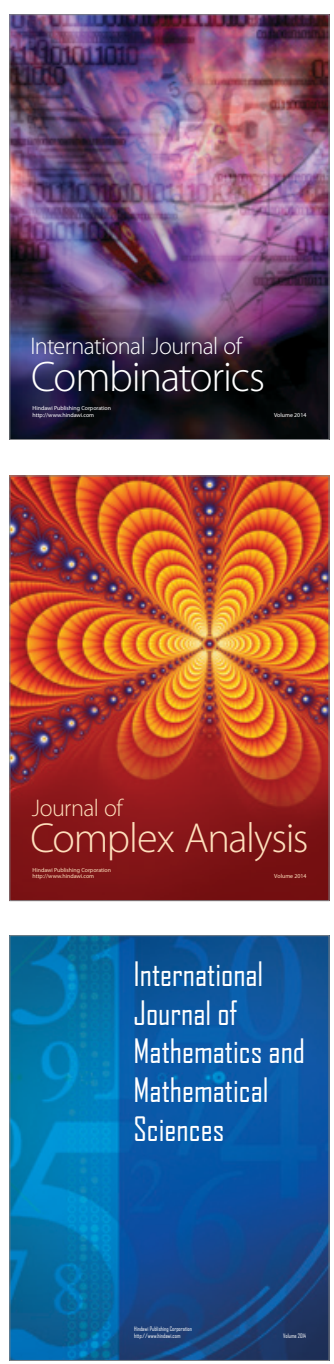
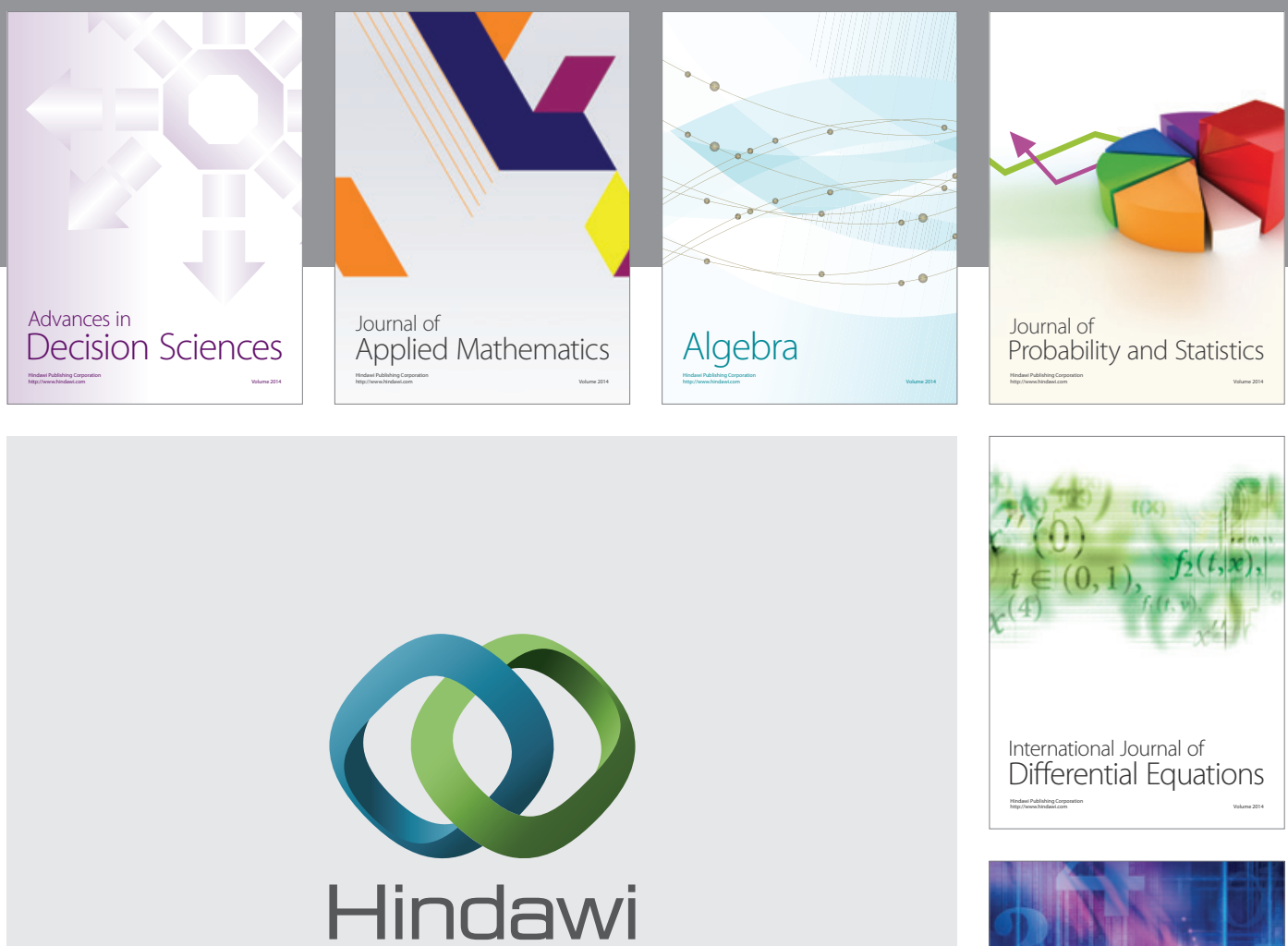

Submit your manuscripts at http://www.hindawi.com
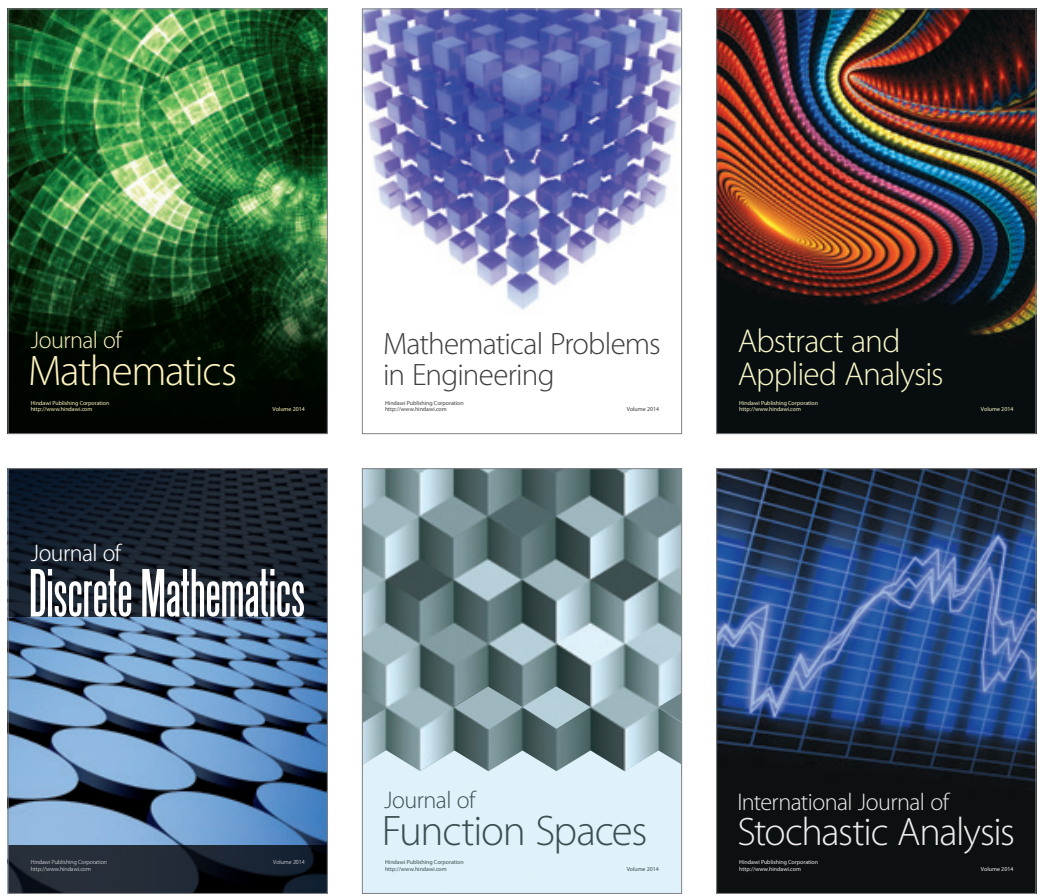

Journal of

Function Spaces

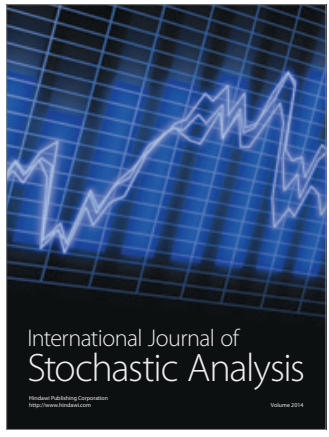

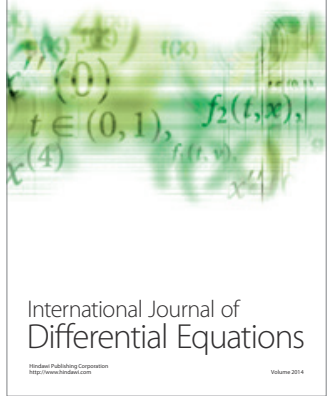
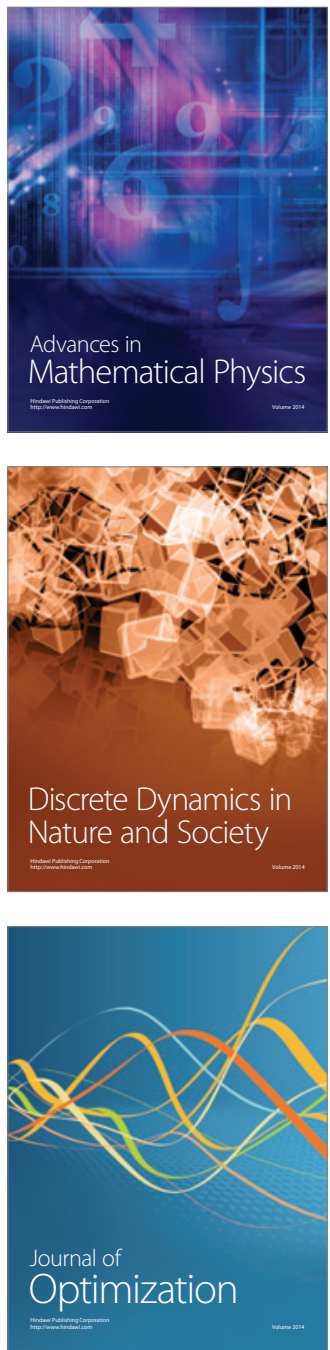